\title{
Recommendations on the interpretation of the new European Medicines Agency Guideline on Bioanalytical Method Validation by Global CRO Council for Bioanalysis (GCC)
}

"These North American and European events provided a unique opportunity for CRO leaders to openly share opinions and perspectives and to agree on unified bioanalytical recommendations specifically in relation with the new EMA guideline."

\begin{abstract}
Mark Boterman', Mira Doigㄹ, Massimo Breda ${ }^{3}$, Steve Lowes ${ }^{4}$, Jim Jersey ${ }^{5}$, Ronald Shoup ${ }^{6}$, Fabio Garofolo ${ }^{7}$, Isabelle Dumont ${ }^{7}$, Suzanne Martinez ${ }^{7}$, Shane Needham ${ }^{8}$, Maria Cruz Caturla ${ }^{9}$, Philippe Couerbe ${ }^{10}$, Joelle Guittard", John Maltas ${ }^{12}$, Tim Lansing ${ }^{13}$, Masood Bhatti ${ }^{14}$, Christine Schieb|15, Petra Struwe ${ }^{15}$, Curtis Sheldon ${ }^{16}$, Roger Hayes ${ }^{17}$, Timothy Sangster ${ }^{18}$, Colin Pattison $^{18}$, Johanne Bouchard ${ }^{19}$, Lee Goodwin ${ }^{20}$, Rafiq Islam ${ }^{21}$, Rudi Segers ${ }^{22}$, Zhongping (John) Lin $^{23}$, Jim Hillier ${ }^{24}$, Wei Garofolo*25, Dieter Zimmer ${ }^{26}$, Lois Folguera ${ }^{27}$, Thomas Zimmermann ${ }^{27}$, Maria Pawula ${ }^{28}$, Marc Moussallie ${ }^{29}$, Leonardo de Souza Teixeira ${ }^{30}$, Thais Rocha ${ }^{30}$, Daniel Tang ${ }^{31}$, Paula Jardieu ${ }^{32}$, James Truog ${ }^{33}$, Jenny Lin ${ }^{33}$, Richard Lundberg ${ }^{34}$, Chris Cox ${ }^{35}$, Alan Breau ${ }^{36}$, Chiara Bigogno $^{37}$, Dick Schoutsen ${ }^{38}$, Carmen Dilger ${ }^{39}$, Mohammed Bouhajib ${ }^{40}$, Ann Levesque ${ }^{41}$, Sofi Gagnon-Carignan ${ }^{41}$, Robert Nicholson ${ }^{42}$, Rand Jenkins ${ }^{42}$, Ming Hung Lin ${ }^{43}$, Shane Karnik ${ }^{44}$, Theo De Boer ${ }^{45}$, Richard Houghton ${ }^{46}$, Rachel Green ${ }^{46}$, William DeMaio ${ }^{47}$, Romuald Sable ${ }^{48}$, Kirk Smith ${ }^{49}$, Christoph Siethoff ${ }^{50}$, Laura Cojocaru ${ }^{51}$, Mike Allen ${ }^{52}$, Tammy Harter ${ }^{53}$, Saadya Fatmi $^{54}$, Farhad Sayyarpour ${ }^{55}$, Michele Malone ${ }^{56}$, Stuart Best ${ }^{57}$ \& Xinping Fang ${ }^{58}$

Note that due to equality principals of Global CRO Council (GCC), authors are presented in alphabetical order of company affiliation.

*Author for correspondence: Global CRO Council (GCC), I5 Sunview Dr, Toronto, Ontario, L4H IY3, Canada Tel.: + I 514236 4225; E-mail: wei@global-cro-council.org; Website: www.global-cro-council.org

Author affiliations can be found at the end of this article
\end{abstract}

Keywords: European Medicines Agency $\approx$ EMA $\approx$ FDA $\approx$ matrix selection $\approx$ reference standards $\approx$ stability

Senior representatives of GCC member companies have thoroughly evaluated and discussed the new European Medicines Agency (EMA; July 2011 [1]) Guideline on Bioanalytical Method Validation (BMV), during the 4th GCC (23 October 2011, Washington DC, USA) and 5th GCC (14 November 2011, Barcelona, Spain) Closed Forums. These North American and European events provided a unique opportunity for CRO leaders to openly share opinions and perspectives and to agree on unified bioanalytical recommendations specifically in relation with the new EMA guideline.

The Global CRO Council for Bioanalysis (GCC) [101] is a global independent group of many Contract Research Organization (CRO) leaders. The GCC was formed in September 2010 to meet regularly and discuss bioanalytical issues and regulatory challenges, many of them unique to the outsourcing industry. The outcome of the discussions held as part of these GCC closed forum meetings are shared with the global bioanalytical community via pertinent publications [2-6] and appropriate conference presentations.

Prior to the issue of this new guideline, the November 2009 Draft EMA Guideline on BMV defined the proposed guideline and criteria of the EMA on validation of bioanalytical methods and their application in the analysis of study samples from animal and human studies [7].

The EMA Guideline states that the validation of the bioanalytical methods to be used on non-clinical pharmacotoxicological studies to be submitted in a marketing authorization application should be performed following the
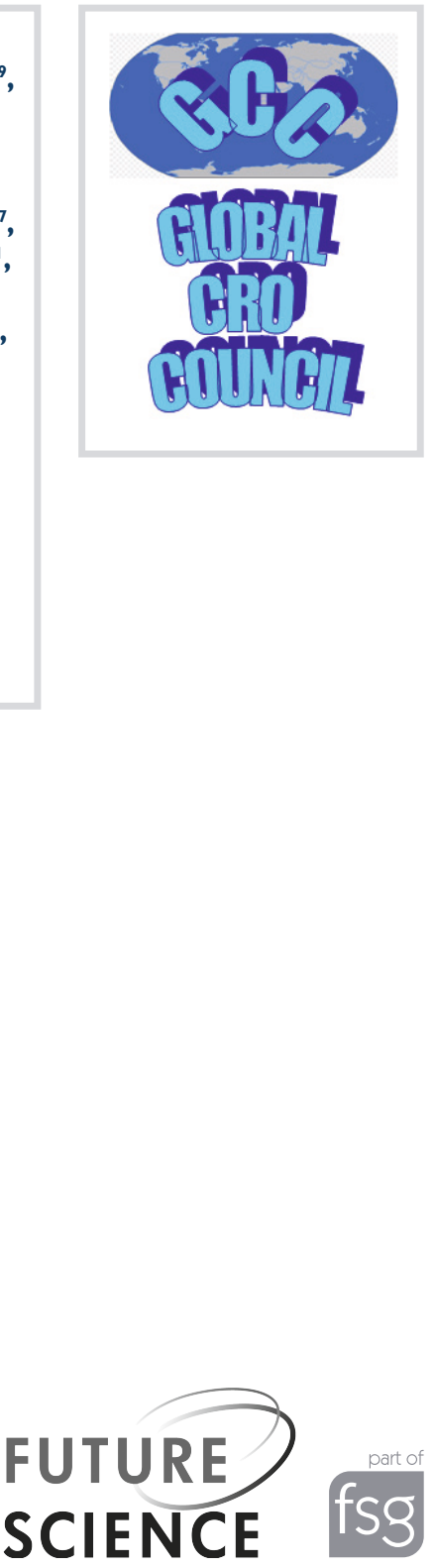
principles of Good Laboratory Practice (GLP) with any aspects of method validation not performed according to GLP clearly identified and their potential impact on the validation status of the method indicated. Additionally, the EMA Guideline states that the validation of bioanalytical methods and the analysis of study samples for clinical trials in humans should be performed following the principles of Good Clinical Practice (GCP). This is in contrast to the 2001 FDA Guidance [8] and will be discussed at a future GCC closed meeting.

The GCC recommendations on the interpretation of the finalized EMA Guideline on BMV were grouped into the following topics:
- Reference Standards: certificates of analysis and internal standards (IS);

- Calibration curve and accuracy;

- IS stability, processed sample stability and matrix effect;

- Analysis of study samples and incurred sample reanalysis (ISR);

- Ligand binding assay (LBA)-specific issues.

Some of the major differences between the finalized EMA Guideline, the 2001 FDA guidance and the Crystal City III White Paper [9] were also presented in a tabular format during the GCC Closed Forums (TABLE I).

\section{Table 1. Some major differences between European Medicines Agency and FDA guidance.}

\begin{tabular}{|c|c|c|c|}
\hline Subject & $\begin{array}{l}\text { European Medicines Agency } \\
\text { Guideline (2011) }\end{array}$ & $\begin{array}{l}\text { US FDA Guidance } \\
(2001)\end{array}$ & US FDA Crystal City III (2007) \\
\hline RFS & $\begin{array}{l}\text { CofA not required for IS } \\
\text { Discusses isotopic purity } \\
\text { expectations of labeled RFS for IS }\end{array}$ & $\begin{array}{l}\text { No reference to } \\
\text { expectations regarding } \\
\text { labeled RFS }\end{array}$ & $\begin{array}{l}\text { No mention of labeled IS. CofA or purity of } \\
\text { IS not always necessary }\end{array}$ \\
\hline Selectivity criteria & $\begin{array}{l}\text { Response of interference is }<20 \% \\
\text { of the LLOQ for analyte and }<5 \% \\
\text { for IS }\end{array}$ & No specific criteria & $\begin{array}{l}\text { Carryover section; the analyte response at } \\
\text { the LLOQ should be at least } 5 \times \text { blank } \\
\text { response }\end{array}$ \\
\hline Selectivity (co-medications) & $\begin{array}{l}\text { Discusses the investigation of } \\
\text { interference from possible } \\
\text { co-administered medications }\end{array}$ & Not discussed & Not discussed \\
\hline Selectivity (metabolites) & $\begin{array}{l}\text { Includes test for possible metabolic } \\
\text { back-conversion }\end{array}$ & $\begin{array}{l}\text { No specific metabolite } \\
\text { tests recommended }\end{array}$ & $\begin{array}{l}\text { Characterization of metabolites should } \\
\text { proceed using a flexible, 'tiered' approach }\end{array}$ \\
\hline LLOQ requirement & $\begin{array}{l}\text { Not higher than } 5 \% \text { of } \mathrm{C}_{\max } \text { in } \mathrm{BE} \\
\text { studies }\end{array}$ & No mention of $C_{\max }$ & Not discussed \\
\hline Calibration curve & $\begin{array}{l}\text { Calibrants stats of all acceptable } \\
\text { runs reported; QCs stats of all runs } \\
\text { reported; truncated curves not } \\
\text { accepted in validation }\end{array}$ & $\begin{array}{l}\text { Reporting of runs and } \\
\text { truncated curves not } \\
\text { discussed }\end{array}$ & $\begin{array}{l}\text { Calibrants and QCs stats of all runs } \\
\text { reported; truncated curves not discussed }\end{array}$ \\
\hline Recovery & Not discussed & Required & Not discussed \\
\hline Incurred sample reanalysis & $\begin{array}{l}\text { Required; number and criteria } \\
\text { provided ( } 10 \% \text { of first } 1000 \\
\text { samples, } 5 \% \text { of the rest) }\end{array}$ & $\begin{array}{l}\text { Not formally discussed in } \\
\text { guidance, but enforced } \\
\text { by the US FDA }\end{array}$ & Recommended \\
\hline Carryover & $\begin{array}{l}\text { Carryover in the blank following the } \\
\text { high standard not greater than } \\
20 \% \text { of the LLOQ and } 5 \% \text { for the IS }\end{array}$ & Not discussed & $\begin{array}{l}\text { LLOQ response at least } 5 \times \text { the response } \\
\text { due to blank matrix. Randomization of } \\
\text { samples to avoid }\end{array}$ \\
\hline Matrix effect & $\begin{array}{l}\text { Includes specifics of evaluation and } \\
\text { criteria (matrix factor) }\end{array}$ & $\begin{array}{l}\text { General statement that it } \\
\text { should be investigated }\end{array}$ & $\begin{array}{l}\text { Includes specifics of evaluation and criteria } \\
\text { (matrix factor) }\end{array}$ \\
\hline Stability & $\begin{array}{l}\text { Provides criteria of } 15 \% \text { from } \\
\text { nominal } \\
\text { In case of a multianalyte BE study, } \\
\text { attention should be paid to stability } \\
\text { of the analytes in the matrix } \\
\text { containing all analytes }\end{array}$ & $\begin{array}{l}\text { No specific criteria; no } \\
\text { mention of multianalyte } \\
\text { studies }\end{array}$ & $\begin{array}{l}\text { No specific criteria; no mention of } \\
\text { multianalyte studies }\end{array}$ \\
\hline Pharmacokinetic outliers & Not recommended & Allowed & Not discussed \\
\hline Dilution integrity & $\begin{array}{l}\text { Includes specifics of evaluation and } \\
\text { criteria (validated by dilution } \\
\text { factors) }\end{array}$ & $\begin{array}{l}\text { Should be demonstrated } \\
\text { by accuracy and } \\
\text { precision parameters in } \\
\text { the validation }\end{array}$ & Not discussed \\
\hline
\end{tabular}




\section{Reference standards: certificates of analysis \& IS}

The new EMA BMV Guideline includes requirements regarding the reference standards used during method validation and analysis of study samples. Section 4.1 of the EMA Guideline states that "A certificate of analysis is required to ensure purity and provide information on storage conditions, expiration date and batch number of the reference standard."

The content and quality of certificates of analysis varies depending on the supplier and, in some cases, certain information, for example expiry or re-test date, is missing. The supplier may sometimes be contacted to obtain the missing information and when this information is not provided some CROs have a general standard operating procedure (SOP) to assign expiry dates to reference standards.

The GCC recommendation for certificates of analysis is shown in Box I.

Section 4.1 of the EMA Guideline also states that "The use of certified standards is not needed for the IS, as long as the suitability for use is demonstrated." The majority of CROs are currently performing tests to demonstrate the suitability of the IS. In general, the interference at the analyte retention time from the presence of the IS is tested in analytical solutions (via the interference check) and in matrix (via selectivity experiments). The suitability of the IS is demonstrated in method development and overall validation results. Additionally, the absence of interference from the IS is usually demonstrated in every analytical batch via blank samples containing only IS (zero sample).

The GCC recommendation for IS is shown in Box 2.

\section{Calibration curve \& accuracy}

Regarding the calibration curve requirements, section 4.1.4 of the EMA Guideline contains a statement that was not present in the draft version of the guideline: "In case all replicates of the $L L O Q$ or the ULOQ calibration standard are rejected then the batch should be rejected from the validation, the possible source of the failure be determined and the method revised (if necessary). If the next validation batch also fails, then the method should be revised before restarting validation." It is a common approach to investigate the cause of the failures before continuing the validation. In general, failures with no assignable cause would prompt the method to go back to the development stage to be re-worked. Different approaches can be followed depending on whether or not there was an assignable cause for calibration standard failure at a limit(s) of quantitation, and dependent on the assay type. Remedial action for assignable causes and investigation of unassignable causes were preferred.

The GCC recommendation for calibration curve is shown in Box 3 .

In the Accuracy section of the EMA Guideline (section 4.1.5), there is a requirement concerning quality control (QC) samples preparation that was also not present in the draft version of the guideline: "The QC samples should be spiked independently from the calibration standards, using separately prepared stock solutions, unless the nominal concentration(s) of the stock solutions have been established." The most common approach in the industry seems to be the use of two separate stocks, unless there is limited compound, and to not determine the nominal concentrations. However, the comparison (correlation) of stock solutions prepared from

\section{Box 1. The GCC recommendation for certificates of analysis.}

A standard format and content for certificates of analysis would be beneficial. However, it is recognized that establishing this standard will not happen prior to the effective date of the Guideline. The minimum information requested by the EMA Guideline reflects the minimum information that the industry would like to receive for reference standards. Therefore, the GCC strongly urges the suppliers of reference standards to provide all of the requested information and recommends that information not supplied is discussed in the bioanalytical report, including an assessment of the potential impact.

\section{Box 2. The GCC recommendation for IS.}

It is recommended to continue performing these tests. Furthermore, the GCC recommends that during method development the threshold of internal standard (IS) concentration for impact on the proposed LLOQ of the method should be determined, for example via an analytical solution interference check, but that no specific experiment determining this is required to be performed during validation. The suitability of the IS in matrix should be assessed during validation with selectivity experiments and continually for all analytical batches using blank samples containing IS. Also, on receipt of each new lot of IS reference standard, an assessment should be made of any information provided and it is recommended that an analytical solution of the IS is used to check for interference. 


\section{Box 3. The GCC recommendation for calibration curve.}

- In the event of calibration standard failure at a LOQ during validation, the GCC recommends the following:

- If all of the LLOQ or ULOQ calibration standards are rejected for one or more validation batches and an assignable cause is determined, then the validation batches should not need to be rejected if the method does not require modification and if the 'model' is not affected. Ligand binding assays using more complex regression models are more likely to be affected by a loss of LOQ and therefore these batches may need to be rejected. If remedial action to correct the assigned cause can be taken, the batches should be accepted and the validation continued. Some evaluations from the affected batches would need to be repeated, for example precision and accuracy at the affected level, selectivity if the LLOQ was affected, and so on;

" If all of the LLOQ or ULOQ calibration standards are rejected for a single validation batch and no assignable cause is determined then the validation batch should be rejected and the validation continued;

" If all of the LLOQ or ULOQ calibration standards are rejected for more than one validation batch and no assignable cause is determined then the validation batches should be rejected and an investigation performed. If necessary, the method should be redeveloped before restarting the validation.

different compound weighings, with an acceptable percent difference of, for example, 5\% between them, is still used.

The GCC recommendation for accuracy is shown in Box 4.

\section{IS stability, processed sample stability \& matrix effect}

Section 4.1.9 of the EMA Guideline mentions the following about stability of the IS: "It is not needed to study the stability of stable-isotope labeled internal standards if it is demonstrated that no isotope exchange reactions occur under the same conditions as the stability of the analyte was demonstrated."It is commonly demonstrated that no isotope exchange reaction occurs and the amount of unlabeled compound is evaluated either in method development, validation and/or with any sample analysis batch. The isotopic purity is not necessarily demonstrated but as previously mentioned, interference coming from the IS is generally evaluated by spiking blank matrix with the IS and evaluating any interfering peak at the analyte's retention time against the LLOQ (specificity) or by injecting replicates of an analytical solution of the IS compared to replicates of the LLOQ (interference check), or a mixture of both tests over the method development/validation and/or sample analysis. When using a new batch of IS, these tests are repeated, generally using the zero sample (i.e., analyte-free sample spiked with IS only) within every analytical batch.

The GCC recommendation for IS stability is shown in Box 5 .

One of the subjects brought forth in the EMA Guideline is the stability of the analyte and the IS in extracts. The EMA Guideline, section 4.1, requires "...stability of the analyte(s) and of the internal standard ... in extracts under the entire period of storage and processing conditions." Postpreparative sample stability following initial injection is normally evaluated, thus effectively assessing the stability of extracts containing both the analyte and the IS.

Furthermore, section 4.1.9. of the EMA Guideline states: “...stability of processed sample ... on-instrument/autosampler stability of the processed sample..." The re-injection reproducibility is performed by re-injecting a whole batch after a period of storage. The processed (postpreparative) sample stability is performed by reinjecting QC samples along with a freshly prepared calibration curve. However, the processed sample stability approach, as described above, arguably demonstrates both extraction stability and re-injection reproducibility.

The GCC recommendation for processed sample stability is shown in Box 6 .

\section{Box 4. The GCC recommendation for accuracy.}

- The GCC recommends that where sufficient reference material is available, independent stock solutions should be used. If sufficient material is not available, this should be discussed within the bioanalytical report. Correlation of stocks prior to use can add extra confidence.

\section{Box 5. The GCC recommendation for IS stability.}

The investigation of stability of stable-labeled internal standard (IS) in solutions should not routinely be performed since IS is only used to compensate for losses during sample processing and is not quantified. Hence, the GCC recommends that there is no need to perform stability of stable-labeled IS in solutions (stock and derived solutions) unless evaluation of the certificate of analysis, pure solution checks or the chemistry of the IS suggests that there is a potential for instability resulting in interference. As previously discussed, the suitability of the stable-labeled IS in matrix should be continually assessed via blank samples containing IS. 


\title{
Box 6. The GCC recommendation for processed sample stability.
}

- GCC recommends that re-injection reproducibility be performed. When an issue is anticipated (e.g., where the method results in a small sample extract volume where re-injection would not be possible), an experiment comparing fresh calibration standards and QC samples with stored extracts can be performed, either in addition or in replace of the re-injection reproducibility experiment. As dry extracts are typically reconstituted in a timely manner, the GCC recommends that the stability of dry extracts would not be required, unless the chemistry of the compound suggests that there is a potential for a stability issue and when it is expected that the dry extracts may be stored prior to reconstitution.

The matrix effect is also a hot topic since the EMA Guideline, section 4.1.8, states: “...the matrix factor (MF) should be calculated for each lot of matrix..."Presently, the MF is used by some in the industry to evaluate the matrix effect. For others, the matrix effect is evaluated by calculating the mean precision and accuracy of plasma concentration values in different matrix lots and by evaluating the suppression/enhancement by comparing responses in the presence and absence of matrix. The use of the matrix factor may not always be applicable, for example because of pure solutions adsorption/solubility issues.

The GCC recommendation for matrix effect is shown in Box 7.

In the same EMA Guideline section, the matrix effect is further discussed as follows: "If $a$ formulation for injection to be administered to the subjects or animals contains excipients known to be responsible for matrix effects, for instance polyethylene glycol or polysorbate, the matrix effects should be studied with matrix containing these excipients... The matrix used for this evaluation should be obtained from subjects or animals administered the excipient, unless it has been demonstrated that the excipient is not metabolized or transformed in vivo." In general, this kind of investigation is not performed and/or it is considered unnecessary, as the impact, if any, is mostly due to the presence of the excipient, not its in vivo metabolism or transformation. Matrix spiked with the excipient is used to investigate potentially problematic excipients since the use of excipientdosed subjects is difficult to obtain in practice, especially in the validation stage. Furthermore, as different formulations could be administered over several studies, it would increase the type of matrices to be assessed for matrix effect.

The GCC recommendation when in presence of excipients known to affect matrix effect is shown in Box 8.

The EMA guideline also recommends that when samples from special populations, for example renally or hepatically impaired populations, are to be analyzed then matrix effects using matrix from such populations should be investigated.

The GCC recommendation for matrix obtained from special populations is shown in Box 9.

\section{Analysis of study samples \& ISR}

The analysis of study samples is described in detail in section 5 of the EMA Guideline. One of the requirements of this section that needs further interpretation was the following: "Before start of the analysis of the study samples the performance of the bioanalytical method should have been verified." It may not be clear how the analytical method should be verified; what period of time needs to elapse before verification is required; if system suitability is considered adequate; or

\section{Box 7. The GCC recommendation for matrix effect.}

\begin{abstract}
As both the FDA Crystal City III White Paper and the EMA Guideline endorse the use of the matrix factor, GCC recommends that this should be the standard approach. It was also recommended that although scientifically the IS-normalized matrix factor should not be required when using a stable-labeled internal standard (IS), the data to calculate this is produced during the matrix effect experiment and, therefore, the presentation of the IS-normalized matrix factor would not require any additional experimental effort. Therefore, the GCC recommends the use of the matrix factor to assess matrix effects, including presenting the IS-normalized data for methods using a stable-labeled IS.
\end{abstract}

\section{Box 8. The GCC recommendation when in presence of excipients known to affect matrix effect.}

Matrix obtained from subjects or animals who had been dosed the excipient are not typically available during validation, and when they are, the use of these samples for validation purposes is not typically discussed within the informed consent or the appropriate clinical or toxicology protocols. Therefore, the GCC recommends the use of matrix spiked with excipient to be used to assess matrix effects during validation. If results or IS response suggests a possible issue during sample analysis, appropriate incurred samples could be investigated, specifically per cause investigation to determine whether or not there is a matrix effect. 


\section{Box 9. The GCC recommendation for matrix obtained from special populations.}

- The GCC recommends that these investigations should not be performed in pre-study validation but within-study to avoid potentially unnecessary investigations. These investigations could be performed by spiking control matrix obtained from special populations (though these matrices may be costly and difficult to source) or by using appropriate incurred samples, for example comparing the analysis of the incurred samples analyzed diluted with normal control matrix and undiluted. However, the use of incurred samples would need to be covered by the informed consent and the clinical protocols.

if a "commissioning batch" should be required. Indeed, this section can be interpreted differently and can refer to system suitability; to a "test batch" containing calibration standards and a varying number of replicates of QC samples (typically informal and not reported); or to a partial re-validation for the re-evaluation of precision and accuracy. Also the period of time could vary widely depending on the interpretation, for example in each batch for system suitability, and from 2 weeks to 2 years for partial re-validation.

The GCC recommendation on analysis of study samples is shown in Box 10.

In section 5.1 of the EMA Guideline it is mentioned that: "For bioequivalence studies ... QC samples should be divided over the run in such a way that the accuracy and precision of the whole run is ensured." In general, half of the QC samples at the front and the other half at the end of the run with all study samples bracketed by the QC samples cannot be considered to be 'divided over the run'. Indeed, QC samples split front and back of an analytical run cannot be adequate to ensure the accuracy and precision of the whole run. Randomization throughout the analytical run can be considered optimal, though bracketing with QC samples may be also acceptable, for small batches or to minimize carryover.

The GCC recommendation on placement of calibration standards and QC samples is shown in Box II.

Regarding the run acceptance criteria described in section 5.2 of the EMA Guideline, it is mentioned that "In case the overall mean accuracy and precision exceeds $15 \%$, this should lead to additional investigations justifying this deviation. In the case of bioequivalence trials it may result in the rejection of the data." During discussions on this section it was pointed out that this is 'study' and not 'run' acceptance criteria and that run acceptance criteria should be adequate.

Meeting these criteria for bioequivalence studies (BE) should be unproblematic as the method used should be robust and bioequivalence studies typically involve a large number of analytical runs. A variety of statistical tests

\section{Box 10. The GCC recommendation on analysis of study samples.}

System suitability is required to determine whether an analytical system is suitable for use on a particular occasion. Verification of the method is used to ensure that a method is performing adequately prior to extraction/analysis of study samples to avoid wasting study samples by using a method that is no longer suitable, for example following a period of non-use, receipt of a new standard and preparation of critical reagents, calibration standards or QC samples, to avoid errors due to incorrectly set up analytical systems and errors due to incorrectly prepared or unsuitable critical reagents, calibration standards and QC samples. Therefore, the GCC recommends that the process and frequency of verification are determined using a risk-based approach, for example if the analytical system set up is known to be problematic or if small sample volumes are available then it would be prudent to make sure the method is working before submitting the study samples for extraction or analysis. Similarly, if a method always performs well and critical reagents, calibrations standards and QC samples are known to be acceptable, then it may not be necessary to verify the method before sample analysis.

- The GCC recommends that verification of an analytical method is discussed in an standard operating procedure (SOP) that contains a definition of how and when to verify an analytical method, including contents of the batch and the acceptance criteria. The verification should be formal and should be a full batch containing samples for the assessment of precision and accuracy. In addition, for LBA an assessment of selectivity/specificity, parallelism and hook effect should also be considered. For ligand binding assays, run(s) of precision and accuracy are assessed as defined by the SOP. The validation acceptance criteria should be applied. The raw data should be retained, and if appropriate the verification should be discussed within the bioanalytical report. To ensure the procedures are followed it may also be prudent to include specific details in the SOP or Study Plan.

\section{Box 11. The GCC recommendation on placement of calibration standards and QC samples.}

- Randomization of QC samples versus minimizing carryover, as well as placement of calibration standards can both be valid and no one rule should be applied to all situations. Therefore, the GCC recommends that placement of calibration standards and QC samples should be determined on a case-by-case basis considering batch size, carryover and so on. 
to determine statistical outliers can be used, for example Grubbs or Dixons Q tests, and then present overall mean and accuracy, including and excluding these statistical outliers. The section 5.2 of the EMA Guideline could be also applied to ligand-binding assays.

The GCC recommendation on acceptance criteria is shown in Box 12 .

It is stated in section 5.5 of the EMA Guideline that "Chromatogram integration and re-integration should be described in a SOP." Having a SOP on chromatogram integration is a general industry practice. However, the contents of the SOP can be very variable, specifically covering how to integrate, how to justify re-integration and how to ensure traceability of integration. An integration SOP should cover all three areas.

The GCC recommendation on chromatogram integration is shown in Box 13 .

Section 6 of the EMA Guideline presents specific information and criteria on ISR. In that section, it is mentioned that "Large differences between results may indicate analytical issues and should be investigated." In evaluating this guideline, high outliers and trends should be taken into consideration, as well as when an investigation and/or reanalysis should be performed. For instance, there is no agreement in the industry on what to consider high outliers since values used may vary between bias greater than 30\% and $100 \%$ from the average.

It seems to be a common approach in the industry, if the ISR acceptance criteria are met, not to investigate any high outliers that did not form a trend. Trends were considered to be a series of results that show commonality, specifically in terms of runs, populations (including gender), subjects or animals, timepoints, dilutions, consistently positive or negative bias (even if ISR acceptance criteria are met), and these should be investigated.

The GCC recommendation on ISR is shown in Box 14 .

\section{LBA: reference standards, selectivity, matrix selection, parallelism \& reagents}

Comparatively to the draft EMA Guideline, the requirements specific to LBA were considerably expanded in the final version of the EMA Guideline, where this topic appears in section 7 with several sub-sections.

Regarding reference standards, section 7.1.1.1 of the guideline states "It is strongly recommended that the batch of the reference standard used for the preparation of the calibration standards and $Q C$ samples is the same as that used for dosing in the non-clinical and clinical studies." It seems this recommendation is already widely followed in the industry.

This is also discussed in the EMA Guideline on the Clinical Investigation of the Pharmacokinetics of Therapeutic Proteins [10], "Contrary to conventional molecules, a pure reference material that can serve as a calibration standard is either difficult or sometimes impossible to obtain for this class of compounds. Therefore extreme care should be taken in order to ensure that the reference material used in the different analytical calibration processes is representative of the material used in clinical trials, including clinical pharmacokinetics."

The GCC recommendation on reference standards is shown in Box 15 .

The EMA Guideline requires in section 7.1.1.3 that "Selectivity should be evaluated at

\section{Box 12. The GCC recommendation on acceptance criteria.}

GCC recommends presenting the overall mean accuracy and precision data with and without proven statistical outliers and widening the criteria to $20 \%$ for ligand binding assay methods. If the overall statistics still fail to meet the acceptance criteria when statistical outliers are omitted from the calculation of the overall statistics, then further investigation would be required, which should be discussed in the bioanalytical report.

\section{Box 13. The GCC recommendation on chromatogram integration.}

- The GCC recommends that the contents of the standard operating procedure discussing integrations should include how to integrate, how to justify re-integration and how to ensure traceability of integration.

\section{Box 14. The GCC recommendation on incurred sample reanalysis.}

\section{The GCC recommends that trends are investigated, including further analysis if appropriate, and that high outliers should not be} investigated or re-analyzed unless they are part of, or form, a trend. 


\section{Box 15. The GCC recommendation on reference standards.}

- The GCC strongly recommends that the same batch of material used for dosing should be made available for bioanalysis. If different batches are used, then this should be discussed in the bioanalytical report.

- For ligand binding assays, comparable performance of each reference standard in the validated assays should be demonstrated. Appropriate analytical design and acceptance criteria should be defined in the standard operating procedure.

the low end of an assay where problems occur in most cases. It may be prudent also to evaluate the selectivity at higher analyte concentrations. In cases where interference is concentration dependent, it is essential to determine the minimum concentrations where interference occurs." It seems that this is routinely assessed at both low and high concentrations or in some cases typically assessed at low concentrations, but additionally assessed at high concentrations when a client requests it. Furthermore, performing a hook effect (prozone effect) experiment would cover the higher concentration.

The GCC recommendation on matrix effect is shown in Box 16.

For matrix selection, the EMA Guideline mentions the possibility to use extracted or alternative matrices in some particular cases. Section 7.1.1.5 of the guideline states the following to that effect: "QC samples should be prepared in the actual sample matrix and the accuracy should be calculated to demonstrate the absence of matrix effect." It is a common approach to prepare QC samples in the actual matrix. This section may be interpreted in different ways, for example including typical matrix effect assessments as well as assessments for use of surrogate matrices.

Moreover, discussions on the interpretation of this section may include when it is acceptable to use surrogate matrices and, as stripped matrices can be variable, whether or not a number of stripped matrices be investigated to ensure conformity against the QC samples prepared in actual sample matrix.

The GCC recommendation on matrix selection is shown in Box 17.
Section 7.1.1.10 of the EMA Guideline discusses parallelism evaluation in LBA: "If study samples are available, parallelism between the calibration standard curve and serially diluted study samples should be assessed (...)" This test is already commonly performed in the industry by assessing parallelism in incurred samples. However, in some cases parallelism assessments are performed only using a spiked sample due to the unavailability of appropriate incurred samples.

Without any doubt, incurred samples are more appropriate for parallelism investigations due to the presence of metabolites, excipients and so on. since the levels of these compounds would vary depending on the timepoint selected. If parallelism in incurred samples is performed during a sample analysis study, rather than a validation study, the subsequent data should be reported in the corresponding bioanalytical report.

The GCC recommendation on parallelism is shown in Box 18.

For the evaluation of the stability of study samples at each temperature at which they will be stored, the EMA Guideline section 7.1.1.11 (part of the ligand binding sections) mentioned that "A bracketing approach may be considered". This approach is also mentioned in the stability section 4.1.9. However, in that same section, the following can be read: "For small molecules it is considered acceptable to apply a bracketing approach ... For large molecules (such as peptides and proteins) stability should be studied at each temperature at which study samples will be stored." Although a bracketing approach is considered acceptable, presently it is common practice

Box 16. The GCC recommendation on matrix effect.

Matrix effect at higher concentrations should be assessed, in particular for ligand binding assays. Therefore, the GCC recommends that matrix effect at low QC and high QC concentrations should be assessed, with an assessment of the prozone effect assessed in one pooled matrix for ligand binding assays.

\section{Box 17. The GCC recommendation on matrix selection.}

The GCC recommends that surrogate matrices should only be used for rare matrices or endogenous analytes. It is recommended to avoid stripped matrices, but if this is the only viable option then the stripped matrix should be treated as a critical reagent and should be assessed each time it is changed. If each stripped matrix is assessed each time it is changed then there would be no requirement to assess a number of stripped matrices during validation, though it may be prudent to perform this during method development to ensure a robust method is developed. 


\section{Box 18. The GCC recommendation on parallelism.}

- The GCC recommends that parallelism should be performed with incurred samples, but care should be taken when selecting the incurred sample with respect to possible metabolites, excipients and so on. If these experiments are performed within a sample analysis study the results should be reported within the bioanalytical report. It is also recommended that the aspects of Good Clinical Practice should be considered. For incurred samples to be used for parallelism evaluations this would need to be included in the clinical protocol and in the informed consent. For pre-clinical studies, the experiment should be included in the toxicology protocol.

for LBA that the actual storage temperature is assessed: stability is performed at, or as close as possible, to the storage temperature of the incurred samples.

A further consideration is that whereas for small molecules proven stability at $-20^{\circ} \mathrm{C}$ would typically indicate acceptability for storage at $-80^{\circ} \mathrm{C}$, for large molecules this would not be the case as lower temperatures may cause stability issues, and vice versa.

The GCC recommendation on stability is shown in Box 19.

Section 7.1.1.12 of the EMA Guideline is related to reagents used in LBA: “...accordingly, when changing reagent batches during validation or sample analysis the analytical performance of the analytical method must be verified to ensure that it is not altered compared with the original or previous batch." It is necessary to clarify up front what are critical reagents and, for specific assays, which of the reagents are considered to be critical.

The GCC recommendation on reagents is shown in Box 20.

\section{Future perspective}

The GCC will continue to provide recommendations on hot topics in bioanalysis of global interest and expand its membership by coordinating its activities with the regional and international meetings held by the pharmaceutical industry. Some suggested future topics for discussion include how to perform matrix effect experiments with respect to hemolysis, hyperlipidemia and excipients, and the GLP conduct of validations. The next GCC Closed Forum is scheduled at the 6th Workshop on Recent Issues in Bioanalysis (6th WRIB) in San Antonio, Texas, USA in March 2012. Please contact the GCC for the exact date and time of the aforementioned meeting, and for all membership information.

\section{Acknowledgements \\ The GCC would like to thank Isabelle Dumont and Suzanne Martinez (Algorithme Pharma, Inc.) and Tammy Harter (Unilabs York Bioanalytical Solutions) for writing the first draft of this White Paper. Wei Garofolo (GCC) for coordinating the logistics of the meetings. The GCC member representatives who facilitated and led the discussion of the chosen topics during the 4 th and 5th GCC meetings. All the member representatives who have sent comments and suggestions to complete this White Paper.}

\section{Financial \& competing interests disclosure} The authors have no relevant affliations or financial involvement with any organization or entity with a financial interest in or financial conflict with the subject matter or materials discussed in the manuscript. This includes employment, consultancies, honoraria, stock ownership or options, expert testimony, grants or patents received or pending, or royalties.

No writing assistance was utilized in the production of this manuscript.

\section{Box 19. The GCC recommendation on stability.}

- For large molecules, if stability was proven at $-20^{\circ} \mathrm{C}$ and at $-80^{\circ} \mathrm{C}$, then the temperatures in between should be acceptable for storage of the incurred samples. Therefore the GCC recommends that for small and large molecules a bracketing approach to stability is acceptable.

\section{Box 20. The GCC recommendation on reagents.}

Formal verification should be performed, similar to the re-verification of an analytical method, and the requirements, content and acceptability should be described in an standard operating procedure, and possibly also in the analytical method or Study Plan. Therefore, the GCC recommends that critical reagents are discussed in an standard operating procedure that contains a definition of critical reagents and how and when to verify a critical reagent, including contents of the batch and the acceptance criteria. A verification batch should typically be a full batch containing samples for the assessment of precision and accuracy and if considered appropriate, samples for the assessment of selectivity/specificity, parallelism and hook effect. The validation acceptance criteria should be applied. The raw data should be retained, and if appropriate the verification should be discussed within the bioanalytical report. To ensure the procedures are followed it may also be prudent to include specific details in the analytical method or Study Plan. 


\section{White PAPER | Boterman, Doig, Breda et al.}

\section{Author Affiliations}

Company names in alphabetical order:

'ABL, Groningen, The Netherlands

${ }^{2}$ ABS Laboratories, Welwyn Garden City, UK

${ }^{3}$ Accelera, Nerviano, Italy

${ }^{4}$ Advion BioServices, Ithaca, NY, USA

${ }^{5}$ Agilux Laboratories, Worcester, MA, USA

${ }^{6} \mathrm{AIT}$ Bioscience, Indianapolis, IN, USA

${ }^{7}$ Algorithme / Simbec, Laval, QC, Canada

${ }^{8}$ Alturas Analytics, Moscow, ID, USA

${ }^{9}$ Anapharm Europe, Barcelona, Spain

${ }^{10}$ Atlanbio, Saint-Nazaire, France

"Avogadro, Toulouse, France

${ }^{12} \mathrm{BASi}$, Kenilworth, Warwickshire, UK

${ }^{13}$ BioAgilytix Labs, Durham, NC, USA

${ }^{14}$ BioPharma Services, Toronto, Ontario, Canada

${ }^{15}$ Celerion, Fehraltorf, Switzerland

${ }^{16}$ Celerion, Lincoln, NE, USA

${ }^{17}$ Cetero Research, Houston, TX, USA

${ }^{18}$ Charles River, Tranent, UK

${ }^{19} \mathrm{CiToxLAB}$, Evreux cedex, France

${ }^{20} \mathrm{Covance}$ Laboratories, Harrogate, UK

${ }^{21}$ EMD Millipore, St Charles, MI, USA

${ }^{22}$ Eurofins, Breda, The Netherlands

${ }^{23}$ Frontage Laboratories, Malvern, PN, USA

${ }^{24}$ Gen-Probe Life Sciences, Livingston, UK

${ }^{25}$ Global CRO Counci

${ }^{26} \mathrm{Harlan}$ Laboratories, Itingen, Switzerland

${ }^{27}$ Harlan Laboratories, Santa Perpetua de Mogoda,

Spain

${ }^{28}$ Huntingdon Life Sciences, Huntingdon, UK

${ }^{29}$ Huntingdon Life Sciences, Somerset, NJ, USA

${ }^{30}$ ICF, Goiania, Brasil

${ }^{31}$ ICON APAC, Shanghai, China

${ }^{32}$ ICON Development Solutions, Whitesboro, NY, USA

${ }^{33} \mathrm{JCL}$ Bloassay USA, Hoffman Estates, Illinois, USA

${ }^{34}$ MEDTOX Laboratories, St Paul, MN, USA

${ }^{35}$ Millipore BioPharma Services, Abingdon, UK

${ }^{36}$ MPI Research, Mattawan, MI, USA

${ }^{37} \mathrm{NiKem}$ Research, Baranzate, Italy

${ }^{38}$ NOTOX, Hertogenbosch, The Netherlands

${ }^{39}$ Nuvisan $\mathrm{GmbH}$, Neu-Ulm, Germany
${ }^{40}$ Pharma Medica Research, Toronto, Ontario, Canada ${ }^{41}$ PharmaNet Canada, Quebec City, Quebec, Canada ${ }^{42}$ PPD, Virginia, USA

${ }^{43}$ Protech Pharmaservices, Taipei City, Taiwan

${ }^{44}$ Pyxant Labs, Colorado Springs, CO, USA

${ }^{45}$ QPS Netherlands BV, Groningen, The Netherlands

${ }^{46}$ Quotient Bioresearch, Fordham, UK

${ }^{47}$ Ricerca Biosciences, Concord, OH, USA

${ }^{48}$ SGS, Wavre, Belgium

${ }^{49}$ Smithers Pharma Services, Wareham, MA, USA

${ }^{50}$ Swiss BioQuant AG, Reinach, Switzerland

${ }^{51}$ Tandem Labs, West Trenton, NJ, USA

${ }^{52}$ Tandem Labs, Durham, NC, USA

${ }^{53}$ Unilabs York Bioanalytical Solutions, York, UK

${ }^{54}$ Warnex Bioanalytical, Laval, Quebec, Canada

${ }^{55}$ WIL Research Company, Ashland, OH, USA

${ }^{56}$ Worldwide Clinical Trials, Austin, TX, USA

${ }^{57}$ Xceleron, York, UK

${ }^{58}$ XenoBiotic Laboratories, Plainsboro, NJ, USA

\section{References}

1 EMA. Guideline on Bioanalytical Method Validation. EMA, Committee for Medicinal Products for Human Use (CHMP), London, UK (2011)

2 Premkumar N, Lowes S, Jersey J et al. Formation of a Global Contract Research Organization Council for Bioanalysis. Bioanalysis 2(11), 1797-1800 (2010).

3 Global CRO council leads to APA opening to CROs! Bioanalysis 3(4), 357 (2011).

4 Lowes S, Jersey J, Shoup R et al. Recommendations on: internal standard criteria; stability; incurred sample reanalysis and recent 483 s by the Global CRO Council for Bioanalysis. Bioanalysis 3(12), 1323-1332 (2011).
5 Breda M. Garofolo F, Caturla MC et al. The 3rd Global CRO Council (GCC) for Bioanalyis at the International Reid Bioanalytical Forum. Bioanalysis 3(24), 2721-2727 (2011).

6 Conference Reports: $4^{\text {th }}$ GCC Closed Forum at AAPS and $5^{\text {th }}$ GCC Closed Forum at EBF - Bioanalysis (2012) (In Press).

7 EMA. Draft Guideline on Validation of Bioanalytical Methods. EMA, Committee for Medicinal Products for Human Use (CHMP), London, UK (2009).

8 US FDA. Guidance for Industry: Bioanalytical Method Validation. US Department of Health and Human Services, US FDA, Center for Drug Evaluation and Research, Rockville, MD, USA (2001).

9 Viswanathan CT, Bansal S, Booth B et al. Quantitative bioanalytical methods validation and implementation: best practices for chromatographic and ligand binding assays. Workshop/Conference Report. AAPS J. 9(1), E30-E42 (2007)

10 EMA. Guideline on the Clinical Investigation of the Pharmacokinetics of Therapeutic Proteins. EMA, Committee for Medicinal Products for Human Use (CHMP), London, UK (2007)

\section{Website}

101 Global CRO Council. www.global-cro-council.org 